\title{
Sum-Capacity of Multiple-Write Noisy Memory
}

\author{
Lele Wang and Young-Han Kim \\ Department of Electrical and Computer Engineering \\ University of California, San Diego \\ La Jolla, CA 92093 USA \\ \{lew001, yhk\}@ucsd.edu
}

\begin{abstract}
Motivated by the emerging interests in non-volatile solid-state computer memories such as flash memories, this paper studies the problem of repeatedly storing information on memory cells with noise and state. The goal is to reliably convey $t$ messages by writing $X_{j}^{n}$ on an $n$-cell noisy memory $p\left(y_{j} \mid x_{j}, y_{j-1}\right)$, which stores $Y_{j}^{n}$ at the $j$-th write. We model this problem as a channel with state and introduce the multiple-write noisy memory model, which includes the write-once memory and flash memory models as special cases. The $t$-write sum-capacity for the multiple-write noisy memory is established as
\end{abstract}

$$
C_{\text {sum }}(t)=\max \left[I\left(X_{1} ; Y_{1}\right)+\sum_{j=2}^{t}\left(I\left(U_{j} ; Y_{j}\right)-I\left(U_{j} ; Y_{j-1}\right)\right)\right]
$$

where the maximum is over all pmfs $p\left(x_{1}\right) \prod_{j=2}^{t} p\left(u_{j} \mid y_{j-1}\right)$ and functions $x_{j}\left(u_{j}, y_{j-1}\right), j=2, \ldots, t$. We derive three outer bounds on the capacity region and discuss their extension to other classes of memory models. These results extend Wolf, Wyner, Ziv, and Körner's work on the binary write-once memory and Fu and Vinck's work on the generalized writeonce memory to noisy memories.

\section{INTRODUCTION AND MAIN RESULT}

A write-once memory (WOM) [12] is a binary information storage medium, where each cell of the memory is initialized at 0 -state and is allowed to change from 0 -state to 1 -state, but not vice versa. Suppose we wish to reliably store $t$ independent messages by writing $t$ times on an $n$-cell WOM. The goal is to maximize the total number of bits stored per cell. One way to deal with this problem is to design codewords such that in any two consecutive writes, there is no transition from 1 to 0 in the same cell position. The total number of codewords satisfying such property characterizes the limit on storing information on the WOM. An underlying assumption in this combinatorial approach is that the memory cell always faithfully store the written codewords.

An alternative (probabilistic) way to attack this problem is through channel coding for communication over a DMC with state. Codewords are assumed to be arbitrary without any constraint, while the memory cell may not always record the input faithfully. Specifically in the WOM, when a cell is at 0 -state, it records what is written. When a cell is at 1-state, it always stores 1 regardless of what the encoder writes (see Figure 1). Clearly, the knowledge of the cell state information help to make the communication more reliable. How much do the encoder and the decoder know about the cell state? The cell state in the WOM is the output of the previous write and before each write, the encoder has access to the whole output sequence, while the decoder does not. Hence, the problem is readily recognized as a channel with state where the state information is available noncausally only at the encoder.

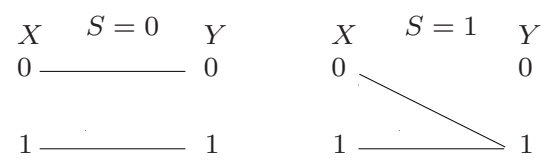

Fig. 1. Binary write-once memory

Many attempts have been made to determine the theoretical limit of maximum number of bits stored per cell. Several code constructions for the binary WOM were proposed by Rivest, Shamir [12] and Cohen, Godlewski, and Merkx [3]. Wolf, Wyner, Ziv, and Körner [13] established the (zero-error) capacity region of the binary WOM. Fiat and Shamir [5] generalized the binary WOM by allowing $q$-ary alphabet and extending the legal state transitions to any directed acyclic graph. This model has been suggested as a good model for flash memories [9]. Practical code constructions based on the generalized WOM have been proposed by Zémor, Cohen [15], Jiang, Bruck [9], Yaakobi, Siegel, Vardy, and Wolf [14] among others. Fu and Vinck [6] established the (zero-error) capacity region of the generalized WOM. Further generalizing the deterministic models for memories, Heegard [8] considered noisy memories and found the capacity region for the WOM with symmetric noise.

The main goal of this paper is to establish the capacity region of noisy memories. Toward this goal, we focus on the memories of the form

$$
\prod_{j=1}^{t} p\left(y_{j} \mid x_{j}, y_{j-1}\right)
$$

where $y_{0}=\emptyset$ by convention. This model is simple, yet still captures the major challenge in updating information on memory cells such as the issue of spatial dependence of the memory state. It is general enough to include all the aforementioned models of WOM, generalized WOM, noisy WOM as special cases.

More specifically, we consider the multiple-write noisy memory (MWNM) model $\left(\mathcal{X}_{1} \times \mathcal{X}_{2} \times \cdots \times \mathcal{X}_{t}\right.$, $\left.p\left(y_{1} \mid x_{1}\right) p\left(y_{2} \mid x_{2}, y_{1}\right) \cdots p\left(y_{t} \mid x_{t}, y_{t-1}\right), \mathcal{Y}_{1} \times \mathcal{Y}_{2} \times \cdots \times \mathcal{Y}_{t}\right)$, as shown in Figure 2.

A $\left(2^{n R_{1}}, \ldots, 2^{n R_{t}}, n\right)$ code for the MWNM consists of

- $t$ message sets $\left[1: 2^{n R_{1}}\right], \ldots,\left[1: 2^{n R_{t}}\right]$,

- $t$ encoders, where encoder 1 assigns a codeword $x_{1}^{n}\left(m_{1}\right)$ to each message $m_{1} \in\left[1: 2^{n R_{1}}\right]$ at the first write and 


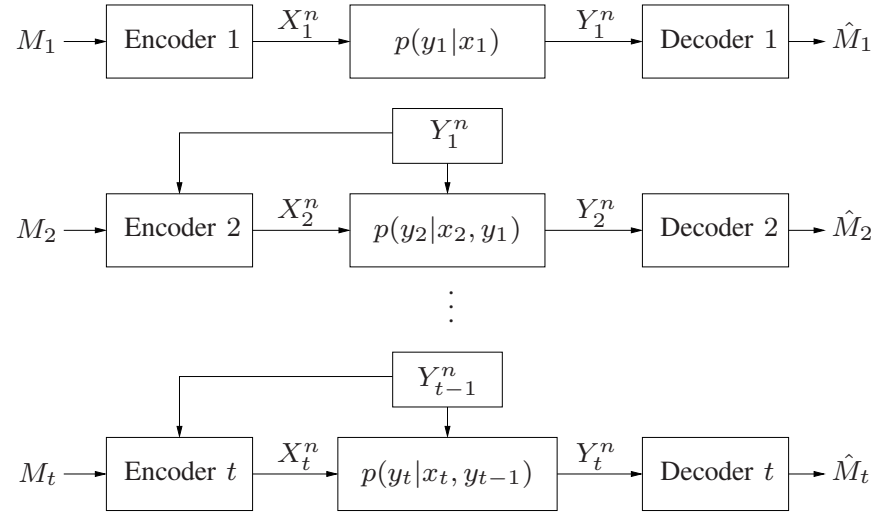

Fig. 2. multiple-write noisy memory

encoder $j \in[2: t]$ assigns a codeword $x_{j}^{n}\left(m_{j}, y_{j-1}^{n}\right)$ to each message $m_{j} \in\left[1: 2^{n R_{j}}\right]$ with side information $y_{j-1}^{n}$ at the $j$-th write, and

- $t$ decoders, where decoder $j \in[1: t]$ assigns an estimate $\hat{m}_{j}$ or an error $e$ to each received sequence $y_{j}^{n}$ at the $j$-th write.

The average probability of error is defined as

$$
P_{e}^{(n)}=\mathrm{P}\left\{\left(\hat{M}_{1}, \ldots, \hat{M}_{t}\right) \neq\left(M_{1}, \ldots, M_{t}\right)\right\} .
$$

A rate tuple $\left(R_{1}, \ldots, R_{t}\right)$ is said to be achievable for the MWNM if there exists a sequence of $\left(2^{n R_{1}}, \ldots, 2^{n R_{t}}, n\right)$ codes such that $\lim _{n \rightarrow \infty} P_{e}^{(n)}=0$. The capacity region $\mathscr{C}$ is the closure of all achievable rate tuples $\left(R_{1}, \ldots, R_{t}\right)$. The $t$ write sum-capacity $C_{\text {sum }}(t)$ of the MWNM is the maximum achievable sum-rate $\sum_{j=1}^{t} R_{j}$.

As the main result of this paper, we will prove the following theorem in Section III.

Theorem 1: The $t$-write sum-capacity of the MWNM is

$$
C_{\text {sum }}(t)=\max \left[I\left(X_{1} ; Y_{1}\right)+\sum_{j=2}^{t}\left(I\left(U_{j} ; Y_{j}\right)-I\left(U_{j} ; Y_{j-1}\right)\right)\right] \text {, }
$$

where the maximum is over all pmfs $p\left(x_{1}\right) \prod_{j=2}^{t} p\left(u_{j} \mid y_{j-1}\right)$ and functions $x_{j}\left(u_{j}, y_{j-1}\right)$ for $j \in[2: t]$.

The achievability proof follows directly by using the Gelfand-Pinsker coding scheme [7]. The main challenges in establishing the sum-capacity lie in the proof of the converse. Note that here the channel state sequence is the output of the previous write and hence may not be i.i.d. over space. Such spatial correlation in the state sequence makes this problem different from the standard setup of channels with discrete memoryless state. However, in our proof of the converse, by carefully combining the standard techniques such as Fano's inequality, Csiszár sum identity [4], and auxiliary random variables identification, we can cancel out such correlation cumulatively. In addition to the sum-capacity, three outer bounds on the capacity region are established in Sections II and III. We discuss their optimality in special cases. The inner and outer bounds recover all existing capacity results.

Heegard [8] considered a more general memory model $\prod_{j=1}^{t} p\left(y_{j}, s_{j} \mid x_{j}, s_{j-1}\right)$, where $s_{j}, x_{j}$, and $y_{j}$ denote the channel state, input, and output at the $j$-th write, respectively.
However, as a cost of generality, it is extremely hard to characterize the capacity region or even the sum-capacity. The capacity region of Heegard's model remains open after more than three decades.

Recently, the phase-change memory (PCM) has received considerable attention as the next generation non-volatile solid-state memory technology. A PCM cell stores data using its amorphous and crystalline states. The cell changes between these two states through high temperature. However, due to the sensitivity of the cells to temperature, it is important to balance the heat in both time and space when programming the cells. Several models have been proposed to study the PCM. Jiang, Bruck, and Li [10] introduced the $(\alpha, \beta, p)$ space-time constrained codes for the PCM, where each segment of $\beta$ cells can be programmed at most $p$ times in any $\alpha$ consecutive writes, which can be viewed as a special case of Heegard's model. As an alternative, Ahlswede and Zhang's model of write-efficient memory [2] has been suggested as a good model for the PCM [11]. In their model, cost functions are introduced based on the assumption that transitions from 0 to 1 or 1 to 0 are costly, while writing the same symbol is cheap. These two models differ from our MWNM model in that the encoder may have only partial access to the state information and that cost functions are introduced. Nonetheless, we will briefly discuss how our results on the MWNM can be applied to the models for phase-change memories in Section IV.

\section{Two-Write MEMory}

We establish three outer bounds on the capacity region. The sum-capacity of the MWNM will be established along the way. For brevity, we first consider the 2-write case in this section and provide its extension to $t$ writes in Section III.

We first recall the following.

Proposition 2.1 (Special case of [8, Theorem 2]): A rate pair $\left(R_{1}, R_{2}\right)$ is achievable for the 2-write MWNM $p\left(y_{1} \mid x_{1}\right) p\left(y_{2} \mid x_{2}, y_{1}\right)$ if

$$
\begin{aligned}
& R_{1} \leq I\left(X_{1} ; Y_{1}\right), \\
& R_{2} \leq I\left(U_{2} ; Y_{2}\right)-I\left(U_{2} ; Y_{1}\right)
\end{aligned}
$$

for some pmf $p\left(x_{1}\right) p\left(u_{2} \mid y_{1}\right)$ and function $x_{2}\left(u_{2}, y_{1}\right)$.

Remark 2.1: This inner bound does not become larger if we evaluate it for general pmf $p\left(x_{1}\right) p\left(u_{2}, x_{2} \mid y_{1}, x_{1}\right)$. To see this, we first note that the inner bound does not become larger if evaluated over $p\left(x_{1}\right) p\left(u_{2}, x_{2} \mid y_{1}\right)$, which follows similar arguments as in the Gelfand-Pinsker coding scheme. Then, it suffices to show for any rate pair $\left(I\left(X_{1} ; Y_{1}\right), I\left(U_{2} ; Y_{2}\right)\right.$ $\left.I\left(U_{2} ; Y_{1}\right)\right)$ attained by $p^{*}\left(x_{1}\right) p^{*}\left(u_{2}, x_{2} \mid y_{1}, x_{1}\right)$, there exist some pmf $\tilde{p}\left(x_{1}\right) \tilde{p}\left(u_{2}, x_{2} \mid y_{1}\right)$ attaining the same rate pair. For any $p^{*}\left(x_{1}\right) p^{*}\left(u_{2}, x_{2} \mid y_{1}, x_{1}\right)$, let $\tilde{p}\left(x_{1}\right)=p^{*}\left(x_{1}\right)$ and

$$
\begin{aligned}
\tilde{p}\left(u_{2}, x_{2} \mid y_{1}\right) & =\sum_{x_{1}} p^{*}\left(u_{2}, x_{2} \mid y_{1}, x_{1}\right) p\left(x_{1} \mid y_{1}\right) \\
& =\sum_{x_{1}} p^{*}\left(u_{2}, x_{2} \mid y_{1}, x_{1}\right) \frac{p\left(y_{1} \mid x_{1}\right) p^{*}\left(x_{1}\right)}{\sum_{x_{1}} p\left(y_{1} \mid x_{1}\right) p^{*}\left(x_{1}\right)} .
\end{aligned}
$$


Then, $p^{*}\left(x_{1}, y_{1}\right)=\tilde{p}\left(x_{1}, y_{1}\right), p^{*}\left(u_{2}, y_{2}\right)=\tilde{p}\left(u_{2}, y_{2}\right)$, and $p^{*}\left(u_{2}, y_{1}\right)=\tilde{p}\left(u_{2}, y_{1}\right)$, which fully determine the achievable rate pair $\left(I\left(X_{1} ; Y_{1}\right), I\left(U_{2} ; Y_{2}\right)-I\left(U_{2} ; Y_{1}\right)\right)$.

We establish three outer bounds on the capacity region.

Proposition 2.2: If a rate pair $\left(R_{1}, R_{2}\right)$ is achievable for the 2-write MWNM $p\left(y_{1} \mid x_{1}\right) p\left(y_{2} \mid x_{2}, y_{1}\right)$, then it must satisfy

$$
\begin{aligned}
R_{1} & \leq I\left(X_{1} ; Y_{1}\right), \\
R_{1}+R_{2} & \leq I\left(X_{1} ; Y_{1}\right)+I\left(U_{2} ; Y_{2}\right)-I\left(U_{2} ; Y_{1}\right)
\end{aligned}
$$

for some $\operatorname{pmf} p\left(x_{1}\right) p\left(u_{2}, x_{2} \mid y_{1}, x_{1}\right)$.

Proof: To establish the first inequality, consider

$$
\begin{aligned}
n R_{1} & =H\left(M_{1}\right) \leq I\left(M_{1} ; Y_{1}^{n}\right)+n \epsilon_{n} \\
& =\sum_{i=1}^{n} I\left(M_{1} ; Y_{1 i} \mid Y_{1, i+1}^{n}\right)+n \epsilon_{n} \\
& =\sum_{i=1}^{n}\left(I\left(M_{1}, Y_{1, i+1}^{n} ; Y_{1 i}\right)-I\left(Y_{1, i+1}^{n} ; Y_{1 i}\right)\right)+n \epsilon_{n} \\
& \stackrel{(a)}{=} \sum_{i=1}^{n}\left(I\left(M_{1}, Y_{1, i+1}^{n}, X_{1 i} ; Y_{1 i}\right)-I\left(Y_{1, i+1}^{n} ; Y_{1 i}\right)\right)+n \epsilon_{n} \\
& \stackrel{(b)}{=} \sum_{i=1}^{n}\left(I\left(X_{1 i} ; Y_{1 i}\right)-I\left(Y_{1, i+1}^{n} ; Y_{1 i}\right)\right)+n \epsilon_{n} \\
& \leq \sum_{i=1}^{n} I\left(X_{1 i} ; Y_{1 i}\right)+n \epsilon_{n},
\end{aligned}
$$

where $(a)$ follows since $X_{1 i}$ is a function of $M_{1}$ and $(b)$ follows since $\left(M_{1}, Y_{1, i+1}^{n}\right) \rightarrow X_{1 i} \rightarrow Y_{1 i}$ form a Markov chain. For the second inequality, the key is to identify $U_{2 i}$ such that $U_{2 i} \rightarrow\left(X_{2 i}, Y_{1 i}\right) \rightarrow Y_{2 i}$ form a Markov chain for each $i \in[1: n]$. Let $U_{2 i}=\left(M_{2}, Y_{2}^{i-1}, Y_{1, i+1}^{n}\right)$. Then

$$
\begin{aligned}
n R_{2} & \leq \sum_{i=1}^{n} I\left(M_{2} ; Y_{2 i} \mid Y_{2}^{i-1}\right)+n \epsilon_{n} \\
& \leq \sum_{i=1}^{n} I\left(M_{2}, Y_{2}^{i-1} ; Y_{2 i}\right)+n \epsilon_{n} \\
& =\sum_{i=1}^{n}\left(I\left(M_{2}, Y_{2}^{i-1}, Y_{1, i+1}^{n} ; Y_{2 i}\right)\right. \\
& \stackrel{(a)}{=} \sum_{i=1}^{n}\left(I\left(M_{2}, Y_{2}^{i-1}, Y_{1, i+1}^{n} ; Y_{2 i}\right)\right. \\
& =\sum_{i=1}^{n}\left(I\left(M_{2}, Y_{2}^{i-1}, Y_{1, i+1}^{n} ; Y_{2 i}\right)\right. \\
& =\sum_{i=1}^{n} I\left(Y_{1 i} ; Y_{2}^{i-1}, M_{2}, Y_{1, i+1}^{n}\right)+I\left(Y_{2 i} ; Y_{2 i}\right)-I\left(U_{2 i} ; Y_{1 i}\right) \\
\left.\quad+I\left(Y_{1 i} ; Y_{1, i+1}^{n}\right)\right)+n \epsilon_{n} &
\end{aligned}
$$

where $(a)$ follows from the Csiszár sum identity. Now combining (1) and (2), we have the bound for sum-rate

$$
\begin{aligned}
& n\left(R_{1}+R_{2}\right) \\
& \quad \leq \sum_{i=1}^{n}\left(I\left(X_{1 i} ; Y_{1 i}\right)+I\left(U_{2 i} ; Y_{2 i}\right)-I\left(U_{2 i} ; Y_{1 i}\right)\right)+n \epsilon_{n} .
\end{aligned}
$$

Using the standard time sharing random variable and taking $n \rightarrow \infty$ completes the proof of Proposition 2.2.

Remark 2.2: The sum-rate outer bound in Proposition 2 together with the inner bound in Proposition 2.1 establishes the 2-write sum-capacity

$$
C_{\text {sum }}(2)=\max \left(I\left(X_{1} ; Y_{1}\right)+I\left(U_{2} ; Y_{2}\right)-I\left(U_{2} ; Y_{1}\right)\right),
$$

where the maximum is over all pmfs $p\left(x_{1}\right) p\left(u_{2} \mid y_{1}\right)$ and function $x_{2}\left(u_{2}, y_{1}\right)$.

Remark 2.3: The outer bound in Proposition 2.2 is tight for any sum-rate $R_{1}+\lambda R_{2}$ with $\lambda \in[0,1]$ and

$$
\begin{aligned}
\max \left(R_{1}\right. & \left.+\lambda R_{2}\right) \\
& =\max \left[I\left(X_{1} ; Y_{1}\right)+\lambda\left(I\left(U_{2} ; Y_{2}\right)-I\left(U_{2} ; Y_{1}\right)\right)\right]
\end{aligned}
$$

where the maximum is over all pmfs $p\left(x_{1}\right) p\left(u_{2} \mid y_{1}\right)$ and functions $x_{2}\left(u_{2}, y_{1}\right)$.

We now present the second outer bound.

Proposition 2.3: If a rate pair $\left(R_{1}, R_{2}\right)$ is achievable for the 2-write MWNM $p\left(y_{1} \mid x_{1}\right) p\left(y_{2} \mid x_{2}, y_{1}\right)$, then it must satisfy

$$
R_{1} \leq I\left(X_{1} ; Y_{1}\right), \quad \text { and } \quad R_{2} \leq I\left(U_{2} ; Y_{2} \mid Y_{1}\right)
$$

for some $p\left(x_{1}\right) p\left(u_{2}, x_{2} \mid y_{1}, x_{1}\right)$.

Proof: The first inequality is exactly the same as in Proposition 2.2. To establish the second inequality, choose $U_{2 i}=\left(M_{2}, Y_{2}^{i-1}, Y_{1, i+1}^{n}, Y_{1}^{i-1}\right)$. Again $U_{2 i} \rightarrow\left(X_{2 i}, Y_{1 i}\right) \rightarrow$ $Y_{2 i}, i \in[1: n]$, as desired. Consider

$$
\begin{aligned}
n R_{2} & \leq I\left(M_{2} ; Y_{2}^{n} \mid Y_{1}^{n}\right)+n \epsilon_{n} \\
& =\sum_{i=1}^{n} I\left(M_{2} ; Y_{2 i} \mid Y_{2}^{i-1}, Y_{1}^{n}\right)+n \epsilon_{n} \\
& \leq \sum_{i=1}^{n} I\left(M_{2}, Y_{2}^{i-1}, Y_{1, i+1}^{n}, Y_{1}^{i-1} ; Y_{2 i} \mid Y_{1 i}\right)+n \epsilon_{n} \\
& =\sum_{i=1}^{n} I\left(U_{2 i} ; Y_{2 i} \mid Y_{1 i}\right)+n \epsilon_{n} .
\end{aligned}
$$

Using standard time sharing random variable and taking $n \rightarrow$ $\infty$ completes the proof.

Remark 2.4: The auxiliary random variable $U_{2}$ identified in Proposition 2.3 is different from the one in Proposition 2.2. As a result, we do not have a nontrivial bound on the sum-rate in Proposition 2.3.

Remark 2.5: The outer bound in Proposition 2.3 is tight when $p\left(y_{j} \mid x_{j}, y_{j-1}\right)$ is deterministic, i.e., the generalized WOM. Taking $U_{2}=Y_{2}$, we have $R_{1} \leq I\left(X_{1} ; Y_{1}\right)=$ $H\left(Y_{1}\right)$ and $R_{2} \leq I\left(U_{2} ; Y_{2} \mid Y_{1}\right)=H\left(Y_{2} \mid Y_{1}\right)$ for some $p\left(x_{1}\right) p\left(x_{2} \mid y_{1}\right)$, which recovers the capacity region of the generalized write-once memories established by $\mathrm{Fu}$ and Vinck [6]. This outer bound follows essentially from the fact that the capacity when side information is available only at the encoder is upper bounded by the capacity when side information is available at both the encoder and the decoder. In the deterministic case, these two capacities coincide.

We now present the third outer bound. 
Proposition 2.4: If a rate pair $\left(R_{1}, R_{2}\right)$ is achievable for the 2-write MWNM $p\left(y_{1} \mid x_{1}\right) p\left(y_{2} \mid x_{2}, y_{1}\right)$, then it must satisfy

$$
\begin{aligned}
R_{1} & \leq I\left(U_{1} ; Y_{1}\right) \\
R_{2} & \leq I\left(U_{2} ; Y_{2} \mid Y_{1}, U_{1}\right) \\
R_{1}+R_{2} & \leq I\left(U_{1} ; Y_{1}\right)+I\left(U_{2} ; Y_{2}\right)-I\left(U_{2} ; Y_{1}\right)
\end{aligned}
$$

for some $p\left(u_{1}, x_{1}\right) p\left(u_{2}, x_{2} \mid y_{1}, u_{1}, x_{1}\right)$.

Proof: In this outer bound, we introduce a new auxiliary random variable $U_{1}$. To establish the first inequality, we expand $Y_{1}^{n}$ differently way from the previous proofs. Identify $U_{1 i}=\left(M_{1}, Y_{1}^{i-1}\right)$. Then

$$
\begin{aligned}
n R_{1} & =\sum_{i=1}^{n} I\left(M_{1} ; Y_{1 i} \mid Y_{1}^{i-1}\right)+n \epsilon_{n} \\
& =\sum_{i=1}^{n}\left(I\left(M_{1}, Y_{1}^{i-1} ; Y_{1 i}\right)-I\left(Y_{1}^{i-1} ; Y_{1 i}\right)\right)+n \epsilon_{n} \\
& \stackrel{(a)}{=} \sum_{i=1}^{n}\left(I\left(U_{1 i} ; Y_{1 i}\right)-I\left(Y_{1, i+1}^{n} ; Y_{1 i}\right)\right)+n \epsilon_{n},
\end{aligned}
$$

where $(a)$ follows from the fact that $\sum_{i=1}^{n} I\left(Y_{1}^{i-1} ; Y_{1 i}\right)=$ $\sum_{i=1}^{n} H\left(Y_{1 i}\right)-H\left(Y_{1}^{n}\right)=\sum_{i=1}^{n} I\left(Y_{1, i+1}^{n} ; Y_{1 i}\right)$.

To establish the second inequality, identify $U_{2 i}=$ $\left(M_{2}, Y_{2}^{i-1}, Y_{1, i+1}^{n}\right)$. Then

$$
\begin{aligned}
n R_{2} & =H\left(M_{2} \mid Y_{1}^{n}, M_{1}\right) \\
& \leq I\left(M_{2} ; Y_{2}^{n} \mid Y_{1}^{n}, M_{1}\right)+n \epsilon_{n} \\
& =\sum_{i=1}^{n} I\left(M_{2} ; Y_{2 i} \mid Y_{2}^{i-1}, Y_{1}^{n}, M_{1}\right)+n \epsilon_{n} \\
& \leq \sum_{i=1}^{n} I\left(M_{2}, Y_{2}^{i-1}, Y_{1, i+1}^{n} ; Y_{2 i} \mid Y_{1 i}, M_{1}, Y_{1}^{i-1}\right)+n \epsilon_{n} \\
& =\sum_{i=1}^{n} I\left(U_{2 i}, Y_{2 i} \mid Y_{1 i}, U_{1 i}\right)+n \epsilon_{n} .
\end{aligned}
$$

Since we use the same $U_{2 i}$ as in Proposition 2.2, the inequality in (2) continues to hold. Combining (3) and (2) establishes the third inequality

$n\left(R_{1}+R_{2}\right) \leq \sum_{i=1}^{n}\left(I\left(U_{1 i} ; Y_{1 i}\right)+I\left(U_{2 i} ; Y_{2 i}\right)-I\left(U_{2 i} ; Y_{1 i}\right)\right)$.

Using the standard time sharing random variable completes the proof of Proposition 2.4.

Remark 2.6: The outer bound in Proposition 2.4 is tight both for any sum-rate $R_{1}+\lambda R_{2}$, where $\lambda \in[0,1]$, and for the deterministic case. Taking $U_{1}=X_{1}$, this outer bound recovers the outer bound in Proposition 2.2. In the deterministic case, this outer bound recovers the outer bound in Proposition 2.3. However, it is not clear whether this is true in general.

\section{Extension to More Than Two Writes}

The inner bound and outer bounds for the 2-write MWNM extend naturally to the general $t$-write MWNM.
Proposition 3.1 (Special case of [8, Theorem 2]): A rate tuple $\left(R_{1}, \cdots, R_{t}\right)$ is achievable for the t-write MWNM $p\left(y_{1} \mid x_{1}\right) \prod_{j=2}^{t} p\left(y_{j} \mid x_{j}, y_{j-1}\right)$ if

$$
\begin{aligned}
R_{1} & \leq I\left(X_{1} ; Y_{1}\right), \\
R_{2} & \leq I\left(U_{2} ; Y_{2}\right)-I\left(U_{2} ; Y_{1}\right), \\
& \vdots \\
R_{t} & \leq I\left(U_{t} ; Y_{t}\right)-I\left(U_{t} ; Y_{t-1}\right)
\end{aligned}
$$

for some pmf $p\left(x_{1}\right) \prod_{j=2}^{t} p\left(u_{j} \mid y_{j-1}\right)$ and functions $x_{j}\left(u_{j}, y_{j-1}\right)$ for $j \in[2: t]$.

Remark 3.1: For a similar reason as in Remark 2.1, this inner bound does not become larger when evaluated for general pmfs $p\left(u_{1}, x_{1}\right) \prod_{j=2}^{t} p\left(u_{j}, x_{j} \mid y_{j-1}, u_{j-1}, x_{j-1}\right)$.

We now extend Proposition 2.2 to the $t$-write MWNM.

Proposition 3.2: If a rate tuple $\left(R_{1}, \cdots, R_{t}\right)$ is achievable for the t-write MWM $p\left(y_{1} \mid x_{1}\right) \prod_{j=2}^{t} p\left(y_{j} \mid x_{j}, y_{j-1}\right)$, then it must satisfy the conditions

$$
\begin{aligned}
R_{1} & \leq I\left(U_{1} ; Y_{1}\right), \\
R_{1}+R_{2} & \leq I\left(U_{1} ; Y_{1}\right)+I\left(U_{2} ; Y_{2}\right)-I\left(U_{2} ; Y_{1}\right),
\end{aligned}
$$

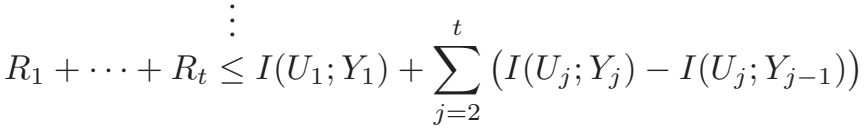

for some $p\left(u_{1}, x_{1}\right) \prod_{j=2}^{t} p\left(u_{j}, x_{j} \mid y_{j-1}, u_{j-1}, x_{j-1}\right)$.

Proof: To bound the sum-rate, the key is still to cancel the nonnegative terms $\sum_{i=1}^{n} I\left(Y_{j-1}^{i-1} ; Y_{j-1, i}\right)$. We play a similar trick as in the proof of Proposition 2.4 to cancel them cumulatively. For each $j \in[2: t]$, identify $U_{j i}=$ $\left(M_{j}, Y_{j}^{i-1}, Y_{j-1, i+1}^{n}\right)$ and assume $Y_{0}^{n}=\emptyset$. We have

$$
\begin{aligned}
& n R_{j} \leq \sum_{i=1}^{n} I\left(M_{j} ; Y_{j i} \mid Y_{j}^{i-1}\right)+n \epsilon_{n} \\
& =\sum_{i=1}^{n}\left(I\left(M_{j}, Y_{j}^{i-1}, Y_{j-1, i+1}^{n} ; Y_{j i}\right)-I\left(Y_{j}^{i-1} ; Y_{j i}\right)\right. \\
& \left.\quad-I\left(Y_{j-1, i+1}^{n} ; Y_{j i} \mid M_{j}, Y_{j}^{i-1}\right)\right)+n \epsilon_{n} \\
& =\sum_{i=1}^{n}\left(I\left(M_{j}, Y_{j}^{i-1}, Y_{j-1, i+1}^{n} ; Y_{j i}\right)-I\left(Y_{j}^{i-1} ; Y_{j i}\right)\right. \\
& =\sum_{i=1}^{n}\left(I\left(M_{j}, Y_{j}^{i-1}, Y_{j-1, i+1}^{n} ; Y_{j i}\right)+I\left(Y_{j-1, i+1}^{n} ; Y_{j-1, i}\right)\right. \\
& \left.\quad-I\left(Y_{j-1, i} ; Y_{j}^{i-1}, M_{j}, Y_{j-1, i+1}^{n}\right)-I\left(Y_{j}^{i-1} ; Y_{j i}\right)\right)+n \epsilon_{n} \\
& =\sum_{i=1}^{n}\left(I\left(U_{j i} ; Y_{j i}\right)-I\left(U_{j i} ; Y_{j-1, i}\right)\right. \\
& \left.\quad+I\left(Y_{j-1, i+1}^{n} ; Y_{j-1, i}\right)-I\left(Y_{j}^{i-1} ; Y_{j i}\right)\right)+n \epsilon_{n} \\
& \stackrel{(a)}{=} \sum_{i=1}^{n}\left(I\left(U_{j i} ; Y_{j i}\right)-I\left(U_{j i} ; Y_{j-1, i}\right)\right. \\
& \left.\quad+I\left(Y_{j-1}^{i-1} ; Y_{j-1, i}\right)-I\left(Y_{j}^{i-1} ; Y_{j i}\right)\right)+n \epsilon_{n},
\end{aligned}
$$

where $(a)$ follows from the fact $\sum_{i=1}^{n} I\left(Y_{j-1, i+1}^{n} ; Y_{j-1, i}\right)=$ $\sum_{i=1}^{n} H\left(Y_{j-1, i}\right)-H\left(Y_{j-1}^{n}\right)=\sum_{i=1}^{n} I\left(Y_{j-1}^{i-1} ; Y_{j-1, i}\right)$. It can be readily observed that the nonnegative terms $\sum_{i=1}^{n} I\left(Y_{j-1}^{i-1} ; Y_{j-1, i}\right), j=2, \ldots, t$, telescope and thus will be canceled in the sum-rate. Using the standard time sharing random variable and taking $n \rightarrow \infty$ completes the proof. 
Remark 3.2: The inner bound in Proposition 3.1 and the outer bound in Proposition 3.2 are tight for sum-rate, which is the main result stated in Theorem 1 .

We now extend Proposition 2.3 to the $t$-write MWNM.

Proposition 3.3: If a rate tuple $\left(R_{1}, \cdots, R_{t}\right)$ is achievable for the t-write MWNM $p\left(y_{1} \mid x_{1}\right) \prod_{j=2}^{t} p\left(y_{j} \mid x_{j}, y_{j-1}\right)$, then it must satisfy the inequalities

$$
\begin{aligned}
R_{1} & \leq I\left(U_{1} ; Y_{1}\right) \\
R_{2} & \leq I\left(U_{2} ; Y_{2} \mid Y_{1}\right), \\
& \vdots \\
R_{t} & \leq I\left(U_{t} ; Y_{t} \mid Y_{t-1}\right)
\end{aligned}
$$

for some $p\left(u_{1}, x_{1}\right) \prod_{j=2}^{t} p\left(u_{j}, x_{j} \mid y_{j-1}, u_{j-1}, x_{j-1}\right)$.

Proof sketch: The proof follows similar arguments as in Proposition 2.3 with $U_{j i}=\left(M_{j}, Y_{j}^{i-1}, Y_{j-1, i+1}^{n}, Y_{j-1}^{i-1}\right)$ for $j \in[1: t]$ and $Y_{0}^{n}=\emptyset$.

Remark 3.3: This bound is tight for the deterministic case. We now extend Proposition 2.4 to the $t$-write MWNM.

Proposition 3.4: If a rate tuple $\left(R_{1}, \cdots, R_{t}\right)$ is achievable for the t-write MWNM $p\left(y_{1} \mid x_{1}\right) \prod_{j=2}^{t} p\left(y_{j} \mid x_{j}, y_{j-1}\right)$, then it must satisfy the inequalities

$$
\begin{aligned}
& R_{1} \leq I\left(U_{1} ; Y_{1}\right), \\
& R_{2} \leq I\left(U_{2} ; Y_{2} \mid Y_{1}, U_{1}\right), \vdots \\
& R_{t} \leq I\left(U_{t} ; Y_{t} \mid Y_{t-1}, U_{t-1}\right), \\
& R_{1}+R_{2} \leq I\left(U_{1} ; Y_{1}\right)+I\left(U_{2} ; Y_{2}\right)-I\left(U_{2} ; Y_{1}\right), \\
& \vdots \\
& R_{1}+\cdots+R_{t} \leq I\left(U_{1} ; Y_{1}\right)+\sum_{j=2}^{t}\left(I\left(U_{j} ; Y_{j}\right)-I\left(U_{j} ; Y_{j-1}\right)\right)
\end{aligned}
$$

for some $p\left(u_{1}, x_{1}\right) \prod_{j=2}^{t} p\left(u_{j}, x_{j} \mid y_{j-1}, u_{j-1}, x_{j-1}\right)$.

Proof sketch: The proof follows similar arguments as in Proposition 2.4 with $U_{j i}=\left(M_{j}, Y_{j}^{i-1}, Y_{j-1, i+1}^{n}\right)$ for $j \in$ $[1: t]$ and $Y_{0}^{n}=\emptyset$.

Remark 3.4: This bound is tight for the deterministic channels and for the sum-rate of arbitrary channels.

\section{Extension to Phase-Change Memory}

In [10], Jiang, Bruck, and $\mathrm{Li}$ introduced the $(\alpha, \beta, p)$ space-time constrained codes for the PCM. Unlike in the MWNM, there is a hidden memory state that is not available at the encoder. Thus, to find the sum-capacity for rewriting on such memories, we look into Heegard's model $\prod_{j=1}^{t} p\left(y_{j}, s_{j+1} \mid x_{j}, s_{j}\right)$, where $S_{1}=\emptyset$ for convention.

Throughout our proof of the converse, the techniques we used are Fano's inequality, Csiszár sum identity, and the chain rule for mutual information, which are valid for Heegard's model as well. The only difference that needs to be checked is whether our identification of auxiliary random variables still guarantees the right Markov chain condition $U_{j i} \rightarrow$ $\left(X_{j i}, S_{j i}\right) \rightarrow\left(Y_{j i}, S_{j+1, i}\right)$ for $i \in[1: n], j \in[2: t]$. This can be verified for all our three identifications. Thus Proposition $3.2-3.4$ can be readily extended to Heegard's general model. In particular, the sum-capacity is upper bounded as

$$
C_{\text {sum }}(t) \leq \max \left[I\left(X_{1} ; Y_{1}\right)+\sum_{j=2}^{t}\left(I\left(U_{j} ; Y_{j}\right)-I\left(U_{j} ; Y_{j-1}\right)\right)\right]
$$

where the maximum is over $p\left(x_{1}\right) \prod_{j=2}^{t} p\left(u_{j}, x_{j} \mid s_{j}\right)$, and lower bounded using the same expression as

$C_{\text {sum }}(t) \geq \max \left[I\left(X_{1} ; Y_{1}\right)+\sum_{j=2}^{t}\left(I\left(U_{j} ; Y_{j}\right)-I\left(U_{j} ; Y_{j-1}\right)\right)\right]$,

except that the maximum is over $p\left(x_{1}\right) \prod_{j=2}^{t} p\left(u_{j}, x_{j} \mid y_{j-1}\right)$. These two bounds can be different in general, however.

In [2], Ahlswede and Zhang proposed the write-efficient memory. They introduced a cost function to characterize whether there is a change in a memory cell. Motivated by this result, we can incorporate a cost function $b(X, S)$ in the MWNM model. By the monotonicity, concavity, and continuity of the capacity-cost function $C_{\text {sum }}(t, B)$ in $B$ and the typicality average lemma [1], our results on the MWNM model can be extended to the sum-capacity with cost constraint $B$ as

$$
\begin{aligned}
C_{\text {sum }}(t, B) & \\
& =\max \left[I\left(X_{1} ; Y_{1}\right)+\sum_{j=2}^{t}\left(I\left(U_{j} ; Y_{j}\right)-I\left(U_{j} ; Y_{j-1}\right)\right)\right],
\end{aligned}
$$

where the maximum is over $p\left(x_{1}\right) \prod_{j=2}^{t} p\left(u_{j}, x_{j} \mid y_{j-1}\right)$ such that $(1 / t) \mathrm{E}\left[\sum_{j=1}^{t} b\left(X_{j}, Y_{j-1}\right)\right] \leq B$.

\section{REFERENCES}

[1] A. El Gamal and Y.-H. Kim. (2010) Lecture notes on network information theory. [Online]. Available: http://arxiv.org/abs/1001.3404

[2] R. Ahlswede and Z. Zhang, "Coding for write-efficient memory," Information and computation, vol. 83, pp. 80-97, 1989.

[3] G. D. Cohen, P. Godlewski, and F. Merkx, "Linear binary code for write-once memories," IEEE Trans. Inform. Theory, vol. 32, no. 5, pp. 697-700, 1986.

[4] I. Csiszár and J. Körner, "Broadcast channels with confidential messages," IEEE Trans. Inform. Theory, vol. 24, pp. 339-348, 1978.

[5] A. Fiat and A. Shamir, "Generalized write-once memories," IEEE Trans. Inform. Theory, vol. 30, no. 1, pp. 470-480, 1984.

[6] F. W. Fu and A. J. H. Vinck, "On the capaicty of generalized writeonce memory with state transitions described by an arbitrary directed acyclic graph," IEEE Trans. Inform. Theory, vol. 45, no. 1, pp. 308313, 1999.

[7] S. I. Gelfand and M. S. Pinsker, "Coding for channels with random parameters," Problem of Control and Information Theory, vol. 9, no. 1, pp. 19-31, 1980.

[8] C. Heegard, "On the capaicty of permanent memory," IEEE Trans. Inform. Theory, vol. IT-31, no. 1, pp. 34-42, 1985.

[9] A. Jiang and J. Bruck, "Joint coding for flash memory storage," in Proc. IEEE International Symposium on Information Theory, Toronto, Canada, 2008, pp. 1741-1745.

[10] A. Jiang, J. Bruck, and H. Li, "Constrained codes for phase-change memories," in Proc. IEEE Information Theory Workshop (ITW), Dublin, Ireland, 2010.

[11] L. A. Lastras-Montano, M. Franceschini, T. Mittelholzer, J. Karidis, and M. Wegman, "On the lifetime of multilevel memories," in Proc. IEEE International Symposium on Information Theory, Seoul, Korea, 2009, pp. 1224-1228.

[12] R. L. Rivest and A. Shamir, "How to reuse a write-once memory," Information and Control, vol. 55, no. 1-3, pp. 1-19, 1982.

[13] J. K. Wolf, A. D. Wyner, J. Ziv, and J. Körner, "Coding for a write-once memory," AT\&T Bell Labs. Tech. J., vol. 63, no. 6, pp. 1089-1112, 1984.

[14] E. Yaakobi, P. H. Siegel, A. Vardy, and J. K. Wolf, "Multiple errorcorrecting WOM-codes," in Proc. IEEE International Symposium on Information Theory, Austin, TX, 2010, pp. 1933-1937.

[15] G. Zémor and G. D. Cohen, "Error-correcting WOM-codes," IEEE Trans. Inform. Theory, vol. 37, no. 3, pp. 730-734, May 1991. 